\title{
What Is the Radiographic Prevalence of Incidental Kienböck Disease?
}

\author{
Wouter F. van Leeuwen MD, Stein J. Janssen MD, \\ Dirk P. ter Meulen MD, David Ring MD, PhD
}

Received: 23 May 2015/Accepted: 24 August 2015/Published online: 1 September 2015

(C) The Association of Bone and Joint Surgeons (R 2015

\begin{abstract}
Background Kienböck disease is characterized by osteonecrosis of the lunate. Not all patients with radiographic evidence of the disease experience symptoms bothersome enough to consult a doctor. Little research has been performed on the prevalence of Kienböck disease, and the prevalence in the asymptomatic population is unclear. Knowledge of the natural course of the disease and how often patients are not bothered by the symptoms is important, because it might influence the decision as to whether disease-modifying treatment would be beneficial.
\end{abstract}

Each author certifies that he or she, or a member of his or her immediate family, has no funding or commercial associations (eg, consultancies, stock ownership, equity interest, patent/licensing arrangements, etc) that might pose a conflict of interest in connection with the submitted article.

All ICMJE Conflict of Interest Forms for authors and Clinical Orthopaedics and Related Research ${ }^{\mathbb{B}}$ editors and board members are on file with the publication and can be viewed on request.

Each author certifies that his or her institution approved the human protocol for this investigation, that all investigations were conducted in conformity with ethical principles of research, and that informed consent for participation in the study was obtained.

Electronic supplementary material The online version of this article (doi:10.1007/s11999-015-4541-1) contains supplementary material, which is available to authorized users.

W. F. van Leeuwen, S. J. Janssen, D. P. ter Meulen, D. Ring Department of Orthopaedic Surgery, Hand and Upper Extremity Service, Massachusetts General Hospital, Harvard Medical

School, Boston, MA, USA

D. Ring $(\bowtie)$

Yawkey Center, Massachusetts General Hospital, Suite 2100,

55 Fruit Street, Boston, MA 02114, USA

e-mail: dring@partners.org
Questions/purposes (1) What is the prevalence of incidental and symptomatic Kienböck disease? (2) What are the factors associated with incidental and symptomatic Kienböck disease? (3) Are there differences in Lichtman stage distribution between incidentally discovered and symptomatic Kienböck disease?

Methods We retrospectively searched radiology reports of all MRI scans, CT scans, and radiographs that included the wrists of 51,071 patients obtained over an 11-year period at one institution to screen for Kienböck disease and avascular necrosis of the lunate. Corresponding MR images, CT scans, or radiographs were reviewed by an orthopaedic hand surgeon to confirm the presence of Kienböck disease when the report was inconclusive. The medical record was reviewed to determine whether the radiographic Kienböck disease was incidental. Prevalences were calculated for both symptomatic and incidental Kienböck disease. Additionally, we assessed the association of age, sex, and race with incidental and symptomatic Kienböck disease as well as the radiographic severity according to the Lichtman classification and calculated odds ratios.

Results We identified 51 cases $(0.10 \%)$ of incidental Kienböck disease and 87 cases $(0.17 \%)$ of symptomatic Kienböck disease out of 51,071 patients. Patients with incidental Kienböck were older (mean, 54 years; SD, 17; mean difference, $-6.1 ; 95 \%$ confidence interval $[\mathrm{CI}],-11$ to $-0.96 ; \mathrm{p}=0.020)$ and patients with symptomatic Kienböck disease were younger (mean, 43 years; SD, 14; mean difference, $5.1 ; 95 \% \mathrm{CI}, 1.2-9.0 ; \mathrm{p}=0.010)$ compared with the group of patients without Kienböck disease (mean, 48 years; SD, 19). Lunate collapse (Lichtman Stages III and IV) was seen in nine of 51 patients (18\%) with incidental Kienböck disease and in 44 of 87 patients (51\%) with symptomatic Kienböck disease (odds ratio, 
$0.21 ; 95 \% \mathrm{CI}, 0.086-0.51 ; \mathrm{p}<0.001)$. Our study did not identify any other factors associated with Kienböck disease.

Conclusions We found that Kienböck disease is diagnosed on radiographs in a notable number of asymptomatic patients and that asymptomatic patients are more likely to have precollapse stages of the disease. This suggests that symptoms and disability do not correlate with pathophysiology, progression, or activity. Patients and surgeons benefit from awareness that symptoms are not a good indicator of the severity or prognosis of pathophysiology and that lunate osteonecrosis can exist with no or insufficient symptoms. This is important when considering treatment, because we cannot distinguish active disease at risk of collapse that could merit disease-modifying treatment from disease that will not progress.

Level of Evidence Level III, prognostic study.

\section{Introduction}

Kienböck disease, or lunatomalacia, was first identified and described by Robert Kienböck (1871-1953) as osteonecrosis of the lunate [17]. The etiology and pathogenesis of the disease are still uncertain, but several mechanical, vascular, and metabolic factors have been proposed [4, 5, 11, 12, 18]. The natural history of Kienböck is incompletely understood [3]. Progression of lunate osteonecrosis can ultimately lead to collapse and fragmentation of the diseased bone with or without secondary distortion of the carpal alignment [2]. What remains uncertain is how frequently the process arrests before complete collapse, the time course of active changes in the lunate, and how often the process arrests at each radiographic stage.

Patients with symptomatic Kienböck disease typically present with a history of wrist pain and/or a decrease in grip strength or wrist motion [9]. Diagnosis and staging of Kienböck disease are based on radiographic imaging. The classification described by Lichtman is most commonly used [1]. However, not all patients with Kienböck disease experience symptoms sufficient to bring the problem to the attention of a physician. In addition, the severity of the disease on radiographs does not always correspond with symptom intensity and magnitude of disability. Kienböck disease is occasionally diagnosed incidentally on radiographs obtained for other indications in patients with no pain or other discrete sources of pain such as a fracture of the distal radius [15].

The prevalence of Kienböck disease in the asymptomatic population is unclear. Mennen and Sithebe [13] studied the radiographs of 1287 asymptomatic African patients and report an incidence of $1.9 \%$. A study by Tsujimoto et al [16] reports a prevalence of $1.2 \%$ in 572 asymptomatic middle-aged and elderly Japanese women. These studies suggest that the symptoms of Kienböck disease are not always sufficient to bring a patient to a doctor. It is counterintuitive that pathophysiology can exist and progress without causing much trouble to a person. Therefore, the unexpectedly high prevalence of incidental, asymptomatic Kienböck disease reminds surgeons that patients with a new diagnosis of Kienböck disease may have disease that has run its course, will not progress, and does not merit potentially disease-modifying treatment.

Our primary study objective was to assess the prevalence of incidental Kienböck disease in patients who underwent an MRI scan, CT scan, or radiograph that included the wrist. We attempted to answer the following questions: (1) What is the prevalence of incidental and symptomatic Kienböck disease? (2) What are the factors associated with incidental and symptomatic Kienböck disease? (3) Are there differences in Lichtman stage distribution between incidental and symptomatic Kienböck disease?

\section{Patients and Methods}

This retrospective study was approved by our institutional review board and a waiver of informed consent was granted. We included radiology reports of all MRI scans, CT scans, and radiographs of the hand, wrist, or forearm performed at our institution between January 1, 2003, and December 31, 2013. We only included patients 18 years of age or older. There were 51,071 patients with a total of 123,566 radiology reports (4824 MRI scans, 2925 CT scans, and 115,817 radiographs). We searched all radiology reports for signs of Kienböck disease. If a patient had more than one imaging test, the disease was considered present if any one of the tests showed signs of lunate avascular necrosis. Staging was based on the first imaging modality demonstrating the disease for each patient. Of the patients not having Kienböck disease, we included the first imaging modality to assemble the reference group.

\section{Outcome Measures and Explanatory Variables}

Our primary outcome measure was presence of incidental Kienböck disease. We identified patients with possible Kienböck disease by searching radiology reports for the following terms: Kienböck, lunatomalacia, and/or the combination of lunate and osteonecrosis. We included common misspellings and synonyms (Appendix 1 
[Supplemental materials are available with the online version of $\left.\mathrm{CORR}^{\circledR}{ }^{\mathbb{1}}\right]$ ). Subsequently, we reviewed all flagged reports to confirm the presence of Kienböck disease. Kienböck disease was defined as radiographic findings consistent with sclerosis or osteonecrosis of the lunate with or without collapse. For 73 radiology reports that were inconclusive, the corresponding MR images, CT scans, or radiographs were reviewed by the senior investigator-an experienced orthopaedic hand surgeon (DR). Patients with other possible causes for radiographic abnormalities of the lunate $(n=66)$ were treated as having no Kienböck disease (eg, postsurgical changes [ $n=14]$, cysts $[n=13]$, degenerative changes $[n=11]$, acute trauma $[\mathrm{n}=9]$, inflammatory processes $[\mathrm{n}=8]$, ulnar impaction [n $=6]$, osteoarthritis [ $\mathrm{n}=2]$, steroid-induced osteonecrosis of the lunate $[n=2]$, and Madelung deformity $[n=1])$. In three cases, observed radiographic lunate abnormalities were absent on followup imaging studies. Additionally, there were five cases in which there remained uncertainty whether radiographic changes of the lunate represented avascular necrosis. These were not considered Kienböck disease. To assess if cases were incidental, all clinic notes of patients identified as having Kienböck disease were reviewed for a patient history of wrist symptoms, a prior diagnosis of Kienböck disease, or other documented wrist abnormalities that could contribute to the abnormal radiographic findings involving the lunate.

The following potential explanatory variables were studied based on prior research [13, 15, 16]: age at the time of imaging, sex, race, and imaging modality.

For all incidental and symptomatic Kienböck cases, staging according to the Lichtman [1] radiographic classification was either extracted from the radiology report or medical record or was determined through evaluation of the radiographs.

\section{Statistical Analyses}

The prevalence of incidental and overall Kienböck disease was calculated and is presented as a percentage of the total number of patients. We presented categorical variables as frequencies with percentages and continuous variables as mean with SD.

In bivariate analyses, differences among the incidental, symptomatic, and combined Kienböck groups were compared with patients with no Kienböck using the Fisher's exact test for sex and the Student's t-test for age. The distribution of lunate collapse (Lichtman Stages III and IV) among incidental and symptomatic patients with Kienböck disease was compared using the Fisher's exact test, and an odds ratio was calculated.
In multivariable logistic regression analyses, we assessed the association of explanatory variables-age, sex, race, and imaging modality-with incidental, symptomatic, and overall Kienböck disease. Odds ratios are provided with $95 \%$ confidence intervals and $\mathrm{p}$ values. A two-tailed $\mathrm{p}$ value $<0.05$ was considered significant. All statistical analyses were performed using Stata ${ }^{\circledR} 13.0$ (StataCorp LP, College Station, TX, USA).

\section{Demographics}

In this study, 51,071 patients were included. Fifty percent was male $(n=25,378)$. The mean age for all patients was 48 years (SD, 19; 95\% confidence interval [CI], 47.7-48.1; standard error, 0.82$)$. The majority of the included patients was white $(76 \% ; \mathrm{n}=38,698)$. Radiographs were made in $95 \%$ of patients $(\mathrm{n}=48,595)$, MRI scans in $3 \%(\mathrm{n}=1682)$, and CT scans in $2 \%(\mathrm{n}=794)$.

\section{Results}

The prevalence for Kienböck disease was $0.27 \%$ (138 of 51,071 ), including $0.10 \%$ for incidental disease (51 of $51,071)$ and $0.17 \%$ for symptomatic disease ( 87 of 51,071). Fifty-one of 138 (37\%) patients had radiographic evidence of Kienböck disease that was diagnosed incidental to the indication for which imaging was ordered. Indications were assessment of fracture after acute trauma $(n=28)$, (osteo)arthritis in the hand or finger arthralgias $(n=16)$, severe flexion contractures in Dupuytren disease $(n=2)$, ligamentous or tendinous injury $(n=2)$, soft tissue mass ( $=1)$, osteomyelitis of the forearm $(\mathrm{n}=1)$, or a painful hand after trigger finger release $(n=1)$. None of these patients had any prior wrist symptoms suggestive of Kienböck disease. The remaining 87 of 138 patients (63\%) were symptomatic or had a previous diagnosis of Kienböck disease.

In multivariable logistic regression analysis, only age was associated with Kienböck disease when stratified into incidental and symptomatic groups, where higher age was associated with greater odds of incidental disease (odds ratio $[\mathrm{OR}], 1.02 ; 95 \% \mathrm{CI}, 1.005-1.04 ; \mathrm{p}=0.010$ ), and higher age was associated with lesser odds of symptomatic disease (OR, 0.99; 95\% CI, 0.97-0.99; $\mathrm{p}=0.034$ ). The Cstatistic was 0.63 and 0.61 for the models assessing factors associated with incidental and symptomatic Kienböck disease, indicating that only a small amount of variation in the prevalence of Kienböck disease is explained by the included variables. Bivariate analysis demonstrated that the mean age was higher in patients with incidental Kienböck 
Table 1. Bivariate analysis of factors associated with Kienböck disease $(\mathrm{n}=51,071)$

\begin{tabular}{|c|c|c|c|c|c|c|c|}
\hline Variable & $\begin{array}{l}\text { Incidental Kienböck } \\
\text { disease }(\mathrm{n}=51) \\
\text { Mean }(95 \% \\
\text { confidence interval })\end{array}$ & $\mathrm{p}$ value* & $\begin{array}{l}\text { Symptomatic Kienböck } \\
\text { disease }(\mathrm{n}=87) \\
\text { Mean }(95 \% \\
\text { confidence interval })\end{array}$ & $\mathrm{p}$ value* & $\begin{array}{l}\text { All Kienböck disease } \\
(\mathrm{n}=138) \\
\text { Mean }(95 \% \\
\text { confidence interval) }\end{array}$ & $\mathrm{p}$ value* & $\begin{array}{l}\text { No Kienböck } \\
\text { disease }(\mathrm{n}=50,933) \\
\text { Mean }(95 \% \\
\text { confidence interval })\end{array}$ \\
\hline \multirow[t]{2}{*}{ Age (years) } & $54(49-59)$ & 0.02 & $43(40-46)$ & 0.01 & $47(44-50)$ & 0.54 & $48(47.7-48.1)$ \\
\hline & Number $(\%)$ & & Number $(\%)$ & & Number (\%) & & Number (\%) \\
\hline Men & $24(44 \%)$ & 0.68 & $49(56 \%)$ & 0.24 & $76(55 \%)$ & 0.23 & $25,302(50 \%)$ \\
\hline \multicolumn{8}{|l|}{ Ethnicity } \\
\hline White & $41(80 \%)$ & 0.66 & $65(75 \%)$ & 0.17 & $106(77 \%)$ & 0.04 & $38,592(76 \%)$ \\
\hline Black & $3(6 \%)$ & & $6(7 \%)$ & & $9(7 \%)$ & & $2978(6 \%)$ \\
\hline Hispanic & $6(12 \%)$ & & $13(15 \%)$ & & $19(14 \%)$ & & $4994(10 \%)$ \\
\hline Asian & $0(0 \%)$ & & $0(0 \%)$ & & $0(0 \%)$ & & $1789(4 \%)$ \\
\hline Other or unknown & $1(2 \%)$ & & $3(3 \%)$ & & $4(3 \%)$ & & $2580(5 \%)$ \\
\hline \multicolumn{8}{|c|}{ Imaging modality (reference: radiograph) } \\
\hline Radiograph & $48(94 \%)$ & 0.45 & $78(90 \%)$ & 0.03 & $126(91 \%)$ & 0.051 & $48,469(95 \%)$ \\
\hline MRI & $3^{\dagger}(6 \%)$ & & $7 *(8 \%)$ & & $10(7 \%)$ & & $1672(3 \%)$ \\
\hline $\mathrm{CT}$ & $0(0 \%)$ & & $2(2 \%)$ & & $2(1 \%)$ & & $792(2 \%)$ \\
\hline
\end{tabular}

* Compared with the no Kienböck disease group; ${ }^{\dagger}$ the three MRI scans in the incidental group were ordered to assess for extensor carpi ulnaris tendinopathy, triangular fibrocartilage complex tear, and chronic forearm ulcers; ${ }^{\ddagger}$ the seven MRI scans in the symptomatic group were second opinions or referred from an outside hospital.

disease $(54$ years; SD, 17; mean difference, $-6.1 ; 95 \% \mathrm{CI}$, -11 to $-0.97 ; \mathrm{p}=0.020)$, lower in the symptomatic Kienböck group (43 years; SD, 14; mean difference, 5.1; 95\% CI, 1.2-9.0; $\mathrm{p}=0.010)$, but did not differ when comparing all Kienböck cases (47 years; SD, 16; mean difference, $0.98 ; 95 \% \mathrm{CI},-2.1$ to $4.1 ; \mathrm{p}=0.54)$ with the reference group (48 years; SD, 19). Furthermore, race differed among the group with all Kienböck cases $(\mathrm{p}=$ 0.039) as compared with the reference group, and imaging modality varied among the symptomatic Kienböck group $(p=0.032)$ as compared with the reference group. None of the patients with Kienböck disease in our cohort were of Asian race (Table 1).

Lunate collapse (Lichtman Stages III and IV) was seen in nine of 51 patients $(18 \%)$ with incidental Kienböck disease and in 44 of 87 patients $(51 \%)$ with symptomatic Kienböck disease (OR, 0.21; 95\% CI, 0.086-0.51; p < 0.001; Table 2). No other factors were found to be associated with symptomatic Kienböck disease.

\section{Discussion}

The natural history of Kienböck disease and the prevalence of incidental Kienböck disease are uncertain. Knowledge of the natural course of the disease and how often patients are not troubled by symptoms is important, because it might influence the decision as to whether disease- modifying treatment would be beneficial. In a large study of images from one institution, we found a prevalence of $0.10 \%$ for incidental Kienböck disease and an overall prevalence of Kienböck disease of $0.27 \%$. Patients with incidental Kienböck disease were older than the reference group and patients with symptomatic Kienböck disease were younger. Lunate collapse (Lichtman Stages III and IV) was more frequently seen in patients with symptomatic Kienböck disease as compared with those with incidental Kienböck disease. It would be helpful to be able to determine when Kienböck disease is still progressing and when it arrests.

This study has a number of limitations. First, because we retrospectively searched radiology reports and did not review all of the imaging studies ourselves, it is possible that earlier stage Kienböck disease has been overlooked by the radiologist in asymptomatic patients. Second, the majority of imaging studies included were radiographs, which do not show Stage I Kienböck disease. Although this is partly offset by the fact that we also searched the subsequent radiographs if patients had more than one within the study period and included reports with the first mention of Kienböck disease and/or lunate osteonecrosis instead of only the first report for every patient, it remains an important limitation, because it risks the potential for increased false-negative findings, especially in the asymptomatic group. Therefore, our estimated prevalence of $0.10 \%$ is conservative and the actual prevalence of 
Table 2. Incidental, symptomatic, and all Kienböck disease per Lichtman stage ( $\mathrm{n}=138)$

\begin{tabular}{llcr}
\hline Lunate collapse & $\begin{array}{l}\text { Incidental Kienböck } \\
\text { disease } \\
\text { Number (\%) }\end{array}$ & $\begin{array}{l}\text { Symptomatic Kienböck } \\
\text { disease } \\
\text { Number (\%) }\end{array}$ & $\begin{array}{c}\text { p value } \\
\text { All Kienböck } \\
\text { disease } \\
\text { Number (\%) }\end{array}$ \\
\hline No collapse (Stages I and II) & $42(30 \%)$ & $43(31 \%)$ & $85(62 \%)$ \\
Collapse (Stages III* and IV) & $9(7 \%)$ & $44(32 \%)$ & $53(38 \%)$ \\
Total & $51(37 \%)$ & $87(63 \%)$ & $138(100 \%)$
\end{tabular}

* Includes Stage IIIa and IIIb Kienböck disease.

Kienböck disease in the asymptomatic population may well be higher. Third, we found that the proportion of MRI scans was larger in patients with Kienböck disease compared with the reference group. However, most of the patients who underwent MRI were referred from an outside hospital to get more sophisticated scans for treatment planning, and prior radiographs showing possible signs of lunate osteonecrosis were made but not available for this study. Consequently, we did not include imaging modality in the multivariable analysis. Additionally, these referrals result in an increased number of symptomatic patients with Kienböck disease in our cohort. Fourth, staging of Kienböck disease was based on the first imaging modality demonstrating the disease, and progression during the study period has not been taken into account. Therefore, the proportion of advanced-stage Kienböck disease (Lichtman Stage III and above) could have been greater if subsequent imaging studies were used for classification.

The prevalence of Kienböck disease in our study $(0.27 \%)$ is considerably less than that reported in previous studies. Mennen and Sithebe [13] found 23 wrists with Kienböck disease in radiographs of 1287 asymptomatic South African patients (1.9\%), whereas Tsujimoto et al [11] found seven cases of Kienböck disease in a cohort of 572 middle-aged and elderly Japanese women (1.2\%). There might be several reasons for this discrepancy. In the present study, radiology reports for 51,071 patients aged 18 years and older with a mean age of 48 years were included, whereas Tsujimoto et al [11] used radiographs of female patients obtained for osteoporosis screening with a mean age of 65 years. The mean age for all patients in the study by Mennen and Sithebe [13], however, is comparable to our cohort. In both studies, the authors reviewed radiographs for a relatively small number of patients. In a study like ours, the best one can do is audit the database by reviewing a manageable number of cases, because it is impossible to do so for all 51,071 patients. By reviewing the borderline cases identified by our initial search and a small set of definitive cases of Kienböck disease, we feel we have made an adequate audit. Additionally, it is possible that the impetus for prior small studies was a spuriously high rate of Kienböck disease and that large databases like ours find lower prevalences.

In our cohort, patients with incidental Kienböck disease were older and patients with symptomatic Kienböck disease younger than patients with no Kienböck disease. The younger, symptomatic patients had more advanced disease. With the caveat that we cannot be certain that incidental Kienböck disease does not advance, these findings are consistent with the concept that Kienböck disease often arrests at a less advanced stage. Relatively minor cases may go undiagnosed and untreated; they accumulate with age resulting in the older average age of patients with incidental Kienböck disease. In contrast, wrists with greater collapse of the lunate may cause more symptoms leading to diagnosis at an earlier age. There is no way of knowing how much wrist pain patients with incidental Kienböck disease had when the disease was active or whether the disease progressed. Few studies have been published on the natural history of Kienböck disease. Kristensen et al [10] report improvement of symptoms in 20 of 26 patients $(77 \%)$ with Kienböck disease that were untreated and in 19 of 23 patients $(83 \%)$ who were treated by immobilization without any signs of radiographic improvement. A study by Mirabello et al [14] also reports that there is poor correlation between clinical and radiographic findings.

In our study, nine of $51(18 \%)$ incidentally diagnosed patients had a collapsed lunate (Stages III and IV), whereas in the study by Mennen and Sithebe [13], of 23 patients, seven had Stage II (30\%), 11 had Stage III (48\%), and five had Stage IV (22\%) Kienböck disease. Four of seven patients with incidental Kienböck disease reported by Tsujimoto et al [16] had Stage II disease (57\%), and three had Stage III (43\%). All 14 patients with incidentally diagnosed Kienböck disease described by Taniguchi et al [15] were at Stage IV. These differences might be the result of interobserver variation in interpretation of the images and staging system. In our study, staging was either extracted from the radiology report or evaluated on imaging studies. Although reported inter- and intraobserver reliability of the Lichtman classification varies among different studies [6-8], this does show that symptoms and 
disability do not tend to correlate with severity of pathophysiology. They also do not seem to correlate well with progression or "activity" of the disease. That is easier to study and quite clear with common diseases such as arthritis. It seems to hold true for Kienböck disease as well as evidenced by the notable prevalence of incidental disease. Patients and surgeons benefit from awareness that symptoms are not always a good indicator of the severity or prognosis of pathophysiology.

If additional research affirms that Kienböck disease is often relatively asymptomatic, that cases of incidental radiographic Kienböck disease accumulate with age, and that incidental cases are associated with relatively less collapse, we might conclude that Kienböck disease often arrests with relatively little collapse. If this is true, it might influence patient and surgeon decision-making. It would mean that at the time of a new diagnosis of Kienböck disease, the patient and surgeon cannot distinguish active disease at risk of collapse that might benefit from disease-modifying treatment and old quiescent disease that will not. Additional longitudinal research with long-term evaluation of patients with incidental Kienböck disease might help us better understand the natural history and determine the proportion of patients that eventually develop symptoms that are disabling enough to consult a doctor. We might also be able to identify factors that predispose patients to collapse of the lunate.

\section{References}

1. Allan CH, Joshi A, Lichtman DM. Kienbock's disease: diagnosis and treatment. J Am. Acad Orthop Surg. 2001;9:128-136.

2. Bochud RC, Buchler U. Kienbock's disease, early stage 3-height reconstruction and core revascularization of the lunate. $J$ Hand Surg Br. 1994;19:466-478.
3. Delaere O, Dury M, Molderez A, Foucher G. Conservative versus operative treatment for Kienbock's disease. A retrospective study. J Hand Surg Br. 1998;23:33-36.

4. Gelberman RH, Bauman TD, Menon J, Akeson WH. The vascularity of the lunate bone and Kienbock's disease. J Hand Surg Am. 1980;5:272-278.

5. Gelberman RH, Salamon PB, Jurist JM, Posch JL. Ulnar variance in Kienbock's disease. J Bone Joint Surg Am. 1975;57:674-676.

6. Goeminne S, Degreef I, De Smet L. Reliability and reproducibility of Kienbock's disease staging. J Hand Surg Eur Vol. 2010;35:555-557.

7. Goldfarb CA, Hsu J, Gelberman RH, Boyer MI. The Lichtman classification for Kienbock's disease: an assessment of reliability. J Hand Surg Am. 2003;28:74-80.

8. Jafarnia K, Collins ED, Kohl HW 3rd, Bennett JB, Ilahi OA. Reliability of the Lichtman classification of Kienbock's disease. J Hand Surg Am. 2000;25:529-534.

9. Keith PP, Nuttall D, Trail I. Long-term outcome of nonsurgically managed Kienbock's disease. J Hand Surg Am. 2004;29:63-67.

10. Kristensen SS, Thomassen E, Christensen F. Kienbock's diseaselate results by non-surgical treatment. A follow-up study. J Hand Surg Br. 1986;11:422-425.

11. Kristensen SS, Thomassen E, Christensen F. Ulnar variance in Kienbock's disease. J Hand Surg Br. 1986;11:258-260.

12. Lluch A, Garcia-Elias M. Etiology of Kienbock disease. Tech Hand Up Extrem Surg. 2011;15:33-37.

13. Mennen U, Sithebe H. The incidence of asymptomatic Kienbock's disease. J Hand Surg Eur Vol. 2009;34:348-350.

14. Mirabello SC, Rosenthal DI, Smith RJ. Correlation of clinical and radiographic findings in Kienbock's disease. J Hand Surg Am. 1987;12:1049-1054.

15. Taniguchi Y, Nakao S, Tamaki T. Incidentally diagnosed Kienbock's disease. Clin Orthop Relat Res. 2002;395:121-127.

16. Tsujimoto R, Maeda J, Abe Y, Arima K, Tomita M, Koseki H, Kaida E, Aoyagi K, Osaki M. Epidemiology of Kienbock's disease in middle-aged and elderly Japanese women. Orthopedics. 2015;38:e14-18.

17. Wagner JP, Chung KC. A historical report on Robert Kienbock (1871-1953) and Kienbock's Disease. J Hand Surg Am. 2005;30:1117-1121.

18. Watson HK, Guidera PM. Aetiology of Kienbock's disease. J Hand Surg Br. 1997;22:5-7. 